must, in a progressively ageing world population, become of even greater importance than they are at present. It provides a long-overdue critical examination of accepted dogma-a 'spring-cleaning' of our thoughts and of the literature on arterial diseases. The facts and arguments, so lucidly presented, invalidate many of the shibboleths used by workers in this field. Thus, for example, dogmatically held views on the relations of fatty streaks to fibrous plaques in arteries are discredited.

The authors are to be congratulated on the directness with which they state their case and views. Whether or not their views are accepted entirely, it is refreshing and helpful, in a field so befogged with hazy thinking, and encumbered by canonical and often obscure statements, to read: "In our view, the thrombogenic concept is the most satisfactory hypothesis [for the genesis of arterial diseases] so far formulated . . ." "There is little doubt that today the emphasis is changing, with thrombosis emerging as the factor of paramount importance in the development of occlusive arterial disease." They suggest that ". . . our knowledge [may be advanced] more rapidly by focusing attention on the circulating blood, rather than on the vessel wall lesions, which may be the result and not the cause of thrombosis"; and finally-"As we have seen, this is a thrombotic disease and, if any relationship does exist between environmental factors and cardiac infarction, we should not try to explain it in terms of 'atherogenesis' but of 'thrombogenesis'."

Full appreciation of this fact-packed, thoughtful and thought-provoking book might, I think, have been facilitated by a five or ten page summary of the main facts and conclusions presented. Also, this analysis would, perhaps, have been enhanced by greater attention to the development of arteries in relation to their diseases and hence by a fuller consideration of the possible roles of arterial remodelling, regeneration and repair in the pathogenesis of occlusive vascular diseases.

Nevertheless, this volume should certainly be of considerable interest to the innumerable pathologists, experimentalists and clinicians who are, to-day, forced to address themselves to this group of diseases, so frequently the cause of premature deaths of mature adults as to constitute the most important man-killer of our time.

\section{T. GILlmaN}

\section{RECENT ADVANCES IN LIPID RESEARCH}

Metabolism and Physiological Significance of Lipids Proceedings of the Advanced Study Course held at Cambridge, September, 1963. Edited by R. M. C. Dawson and D. N. Rhodes. Pp. ix +657 . (London, New York and Sydney: John Wiley and Sons, Ltd., 1964.) 147s.

$M^{E T A B O L I S M}$ and Physiological Significance of M Lipids comprises the collected papers presented at an advanced study course on "Lipids" held in Cambridge in September, 1963. The contributors include many of the world's leading workers in this field of biochemistry. The range of topics discussed and the wealth of detailed information which the book contains indicate clearly both the enormous increase in interest in the biochemistry and physiology of lipids, which has taken place especially in the past two decades or so, and also the challenging problems at present confronting research workers. It is evident that the advances in our knowledge of lipids with which this volume is concerned owe much to the development of modern techniques, particularly to chromatographic procedures and to the use of isotopic tracer methods for the elucidation of metabolic pathways in tissues.

In addition to the two major sections on metabolism and on the physiological significance of lipids, shorter specialized sections deal with lipid absorption, lipids of the nervous system and the association of lipids and proteins in biological systems. Brief reports of the contributions to two symposia, one on the fatty acid composition of lipids and enother on techniques, are also included.

In the section on metabolism of lipids, the biosynthesis and inter-conversion of fatty acids in animal and plant tissues are discussed by several contributors. In animal tissues de novo synthesis from acetyl $\mathrm{CoA}$ and malonyl $\mathrm{CoA}$, elongation and desaturation reactions all seem to be involved; in plant tissues there is no direct evidence at present of elongation of palmitate to stearate or of conversion of the latter acid to oleate.

The synthesis of characteristic branched-chain fatty acids by mycobacteria is also discussed. Other papers in this section deal with recent work on the biosynthesis of sterols, triglycerides and phospholipids. The metabolism of phosphoinositides is described and a hypothetical scheme for their possible role in the transport of cations across membranes is presented. Lipases and phospholipases are the subjects of several contributions, and work on the biochemical characterization of these enzymes is reviewed; the recognition of phospholipase activity in tissues and of the enzymic re-acylation of lysophosphatides has raised the question of the physiological significance of these enzymes in the selective turnover of fatty acids in tissue phospholipids.

In papers dealing with lipid absorption, recent advances in the physicochemical aspects of fat digestion and the mechanisms of transport of lipid material across the intestinal mucosa are reviewed; differences are reported in the mode of transport between different species and between different strains of rats.

Papers on the physiological significance of lipids cover a wide variety of problems. The role of plasma triglycerides in fat transport, the metabolism of short- and long-chain free fatty acids of plasma by various species and the difficult question of the part played by lipids in blood coagulation are reviewed. In other papers in this section lipid metabolism in ruminants and the biosynthesis of milk lipids are considered. Fatty infiltration of the liver as a result of nutritional deficiency or following exposure to toxic agents is discussed in two articles and the hypothesis is proposed that inhibition of lipoprotein formation plays a part in the development of fatty liver. Several contributions are orientated towards the more physicochemical aspects of lipids as membrane components, and others deal with the effects of drugs and hormones on lipid metabolism. In a paper on the lipids of bacterial membranes, the isolation of amino-acid esters of phosphatidylglycerol from a number of microorganisms is described and their physiological function in bacterial membranes is considered.

Lipids of the nervous system are the subject of four contributions which cover biosynthetic mechanisms, the presence of lipolytic enzymes in nervous tissue and their possible significance in demyelination, a review of lipid motabolism in myelin and the lipidoses.

The section on lipid-protein associations includes an extensive and stimulating discussion of the part played by lipids in mitochondrial function.

Those responsible for the organization of this conference on lipids and the editing of its proceedings deserve great praise for their part in producing this excellent collection of authoritative articles. The book is well produced and will be invaluable, not only to biochemists but also to scientists in other disciplines whose interests involve lipids. Each paper includes a useful bibliography together with a summary of the audience discussion which followed its presentation. In view of the great amount of information the book contains and because some aspects of lipid biochemistry are discussed from different points of view by several of the contributing authors, a rather more detailed index would have been an advantage.

G. R. WeHSTER 